\title{
Passivity Properties of Low-Sensitivity Digital Filter Structures
}

\author{
P. P. VAIDYANATHAN, MEMBER, IEEE, AND SANJIT K. MITRA, FELLOW, IEEE
}

\begin{abstract}
A general representation of a class of low passband sensitivity digital filter structures is proposed. The proposed representation for a transfer function of order $N$ consists of an $(N+1)$-pair memoryless system terminated at $N$-pairs by delays. The $(N+1)$-pair system contains only adders and multipliers, and is described by an orthogonal transfer matrix. The set of terminating delays can be looked upon as an $N$-pair system with transfer matrix $z^{-1} 1$. Certain wave digital filter structures, Gray-Markel lattice structures and the coupled-form biquadratic section belong to the general form advanced here. Several properties satisfied in these special cases are derived in a unified manner using the generalized representation. Also, a quantization scheme that makes the structure free from zero-input limit cycles even under time-varying conditions is advanced, unifying similar such results independently reported for the above well-known structures.
\end{abstract}

\section{INTRODUCTION}

$I^{N}$ $\mathrm{N}$ THE literature on digital filtering, a number of papers have drawn attention to the importance of obtaining structures that perform satisfactorily under finiteprecision constraints [1]-[9]. The importance of passivity concept in this regard has been identified in a number of these contributions. For example, wave digital filters [2], [3], well known for low-sensitivity properties, satisfy certain passivity conditions [4]. ${ }^{1}$ Likewise, the orthogonal digital filters, introduced by DeWilde and Deprettere [5], and the Gray and Markel structures [6], [7], are again known to have inherent passivity properties [9]. A general framework for low-sensitivity digital filters, based on the concepts of "bounded real" (BR) and "lossless bounded real" (LBR) functions and matrices has been recently advanced [10], [11]. The LBR property is essentially the characterizing feature of the well-known scattering matrices of lossless continuous-time multiport networks [12]-[14], and hence, plays a critical role in the synthesis of passive filters.

In an interesting development due to Barnes and Fam [15], it has been shown that state-space realizations of digital filters with a "minimum-norm" state transition matrix can be made free from overflow oscillations that are normally caused by quantization nonlinearities. Indeed, these structures also satisfy a passivity requirement. Thus

Manuscript received November 7, 1983; revised June 28, 1984. This work was supported in part by the National Science Foundation under Grant ECS 82-18310, and in part by Grant ECS 84-04245.

P. P. Vaidyanathan is with the Department of Electrical Engineering, California Institute of Technology, Pasadena, CA 91125.

S. K. Mitra is with the Department of Electrical and Computer Engineering, University of California, Santa Barbara, CA 93106.

${ }^{1}$ Fettweis [20] has also advanced and studied the concepts of pseudopassivity and pseudolosslessness in certain generalized signal flow networks. the "minimum-norm" state transition matrix of a stable minimal system is a BR (rather than LBR) matrix. (Indeed, if the state transition matrix were LBR, i.e., orthogonal, then all its eigenvalues would lie on the unit circle, implying an unstable system.) Barnes and Fam also point out that the coupled form digital filter structure is a secondorder minimum-norm system. In [9], Gray again draws attention to the passivity properties of the coupled-form structure, and proceeds to establish a number of results concerning suppression of limit cycles. In his earlier developments, Fettweis [4], [16] establishes the crucial role of passivity for limit-cycle suppression in digital filters. (In fact, the orthogonal filter structures of Gray and Markel are known to be a special form of wave filters [17].)

The main purpose of this paper is to derive these and new results in a unified manner, based on a general representation of the digital filter structures concerned. Among the issues considered here are certain kinds of stability properties under time-varying conditions, certain possibilities of avoiding parasitic oscillations (due to arithmetic rounding) and overflow under time-varying conditions, and some orthogonality relations. These properties are derived making use only of the property of "lossless bounded reality."

\section{THE GenERAL Framework}

We begin with a review of definitions. Let $\mathscr{T}(z)$ be a discrete-time transfer matrix defined by

$$
\boldsymbol{Y}(z)=\mathscr{T}(z) \boldsymbol{U}(z)
$$

wherc $U(z)$ and $Y(z)$ are $Z$-transforms of input and output vectors, respectively. $\mathscr{T}(z)$ is called bounded real (BR) if (a) $\mathscr{T}(z)$ is real for real $z$, (b) Each entry of $\mathscr{T}(z)$ is stable, i.e., has all poles in $|z|<1$, and (c) $\mathscr{T}^{\dagger}\left(e^{j \omega}\right) \mathscr{T}\left(e^{j \omega}\right)$ is bounded above by the identity matrix 1 for all $\omega$, i.e.,

$$
\boldsymbol{V}^{\dagger} \mathscr{T}^{\dagger}\left(e^{j \omega}\right) \mathscr{T}\left(e^{j \omega}\right) \boldsymbol{V} \leqslant \boldsymbol{V}^{\dagger} \boldsymbol{V}, \quad \text { for all } \boldsymbol{V} .
$$

Here superscript dagger $\dagger$ denotes transposed conjugation. The matrix $\mathscr{T}(z)$ is called lossless bounded real (LBR) if, in addition, (2) holds with equality for all $\omega$, i.e., if $\mathscr{T}^{\dagger}\left(e^{j \omega}\right) \mathscr{T}\left(e^{j \omega}\right)=\mathbf{1}$ for all $\omega$. Thus for an LBR system,

$$
\boldsymbol{Y}^{\dagger}\left(e^{j \omega}\right) \boldsymbol{Y}\left(e^{j \omega}\right)=\boldsymbol{U}^{\dagger}\left(e^{j \omega}\right) \boldsymbol{U}\left(e^{j \omega}\right)
$$

for all inputs $U$ and all $\omega$. In the time domain, the LBR 
property translates to

$$
\sum_{n \rightarrow 0}^{\infty} \boldsymbol{y}^{\prime}(n) \boldsymbol{y}(n)=\sum_{n=0}^{\infty} \boldsymbol{u}^{\prime}(n) \boldsymbol{u}(n)
$$

for all finite energy inputs $\{\boldsymbol{u}(n)\}$, assuming zero initial conditions. For a BR system, inequalitites of the form $\leqslant$ replace the equalities in (3) and (4).

For the case of single-input, single-output systems, a BR transfer function $H(z)$ satisfies $|H(z)|<1$ for all $|z|>1$ unless $H(z)$ is a constant equal to \pm 1 . (In addition, if $H(z)$ is allpass, i.e., LBR, it can be shown that $|H(z)|>1$ for $|z|<1$.) Note that each entry of a BR transfer matrix is individually BR. A structurally lossless (passive) system is one which satisfies the LBR (BR) property regardless of the values of the actual parameters characterizing the system, as long as they remain in a certain range.

The wave digital cascade filters and the Gray-Markel lattice structures are based on LBR two-pair building blocks ${ }^{2}$ (or their denormalized versions) and many of their properties derive from the paraunitary property of an LBR system, namely, $\mathscr{T}^{t}\left(z^{-1}\right) \mathscr{T}(z)=\mathbf{1}$ for all $z$. In addition, wave digital filters, Gray-Markel lattices and the coupledform structure can all be represented in a unified manner in the form shown in Fig. 2 where $\mathscr{T}$ is a memoryless $(N+1)$-pair digital structure containing only multipliers and adders. Appendix B includes a proof of this statement. In the case of wave filters, $\mathscr{T}$ can be assumed bounded real without any loss of generality, as wave filters can be normalized if necessary for this purpose. By connecting $N$ of the terminal pairs with delays (which are LBR elements) an $N$ th-order BR function $H(z)=Y(z) / U(z)$ is obtained. In the case of (normalized) Gray-Markel structures, $\mathscr{T}$ is actually LBR, and the constraining of its $N$ terminal pairs by a diagonal LBR matrix of delays gives rise to an $N$ th-order all-pass function $H(z)$. Finally in the case of the coupled form $N=2$ and $\mathscr{T}$ is strictly lossy BR for poles inside the unit circle and LBR for poles on the unit circle [9], [15]. (In fact for the coupled form, we ignore the presence of the input $U$ and output $Y$. The two-pair obtained by extracting both the delays is designated $\mathscr{T}$. The actual system input and output could be anywhere inside, and, in general, the transfer function does not constitute a BR function.)

Before analyzing the structure of Fig. 2, we make the following observation: If an $(N+M)$-pair LBR structure is constrained at its $N$ terminal pairs by an $N$-pair BR structure, the resulting $M$-pair is BR. Moreover, if the constraining $N$-pair is LBR, it results in an LBR $M$-pair. Note also that, unlike PR (positive real) matrices [14], a BR transfer matrix need not be a square matrix, and can, in general, be an $R$-input, $M$-output system. Thus the concept of "ports" is no longer necessary for the BR case. For the rest of the paper, however, we shall consider only square BR matrices.

\footnotetext{
${ }^{2}$ In Appendix A, an LBR two-pair is reviewed, where a digital two-pair is a two-input two-output systems, as shown in Fig. 1.
}
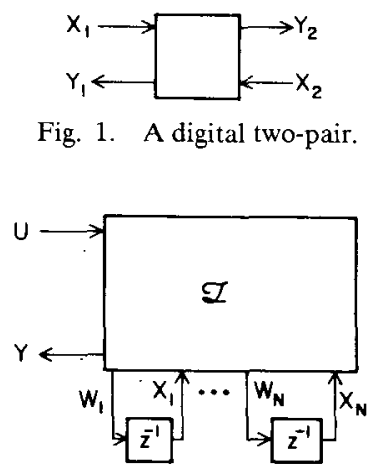

Fig. 2. The unified representation.

\section{Properties of the General Representation}

For generality, let us assume that the $(N+1)$-pair $\mathscr{T}$ shown in Fig. 2 is time-varying, but at each sampling instant satisfies $\mathscr{T}^{t}(n) \mathscr{T}(n) \leqslant 1$. The time-invariant case is clearly included as a special case. We then have,

$$
y^{2}(n)+\sum_{i=1}^{N} w_{i}^{2}(n) \leqslant u^{2}(n)+\sum_{i=1}^{N} x_{i}^{2}(n) .
$$

We also assume for the present that all arithmetic is performed with infinite precision and infinite dynamic range. Observing that $x_{k}(n)$ is a delayed version of $w_{k}(n)$, and summing (5) from 0 to $M$ ( $M$ being any integer) we get

$$
\sum_{n=0}^{M} y^{2}(n) \leqslant \sum_{n=0}^{M} u^{2}(n)+\sum_{i=1}^{N} x_{i}^{2}(0)-\sum_{i=1}^{N} x_{i}^{2}(M+1)
$$

If $\mathscr{T}$ is actually LBR, the inequality in (6) becomes an equality and we have the following power balance equation:

Total output encrgy from $n=0$ to $n=M$

$$
\begin{aligned}
= & {[\text { Total input energy from } n=0 \text { to } n=M] } \\
& +[(\text { initial stored energy at } n=0) \\
& -(\text { energy stored at } n=M+1)] .
\end{aligned}
$$

Moreover, from (5) we have for the lossless case

$$
y^{2}(n)=u^{2}(n)+\sum_{i=1}^{N} x_{i}^{2}(n)-\sum_{i=1}^{N} x_{i}^{2}(n+1) .
$$

Thus the "instantaneous output energy" is precisely the "instantaneous input energy" minus the instantaneous increase in internal energy. The input $u(n)$ is the only source of energy and $y(n)$ is the only means of energy extraction. If $\mathscr{T}$ is BR rather than LBR, then, in addition to the loss of energy via $y^{2}(n)$, there is also a loss of energy inside the structure resulting in an inequality rather than equality in (8).

The results regarding stability, derived in [7] for infinite precision arithmetic, under time-varying conditions also hold under the above general setup. For example, a finite bound on the energy of the input sequence implies a finite bound on the energy of the output sequence, establishing 
"finite energy stability." Similarly, "bounded mean-square stability" [7] also follows from (6).

It is possible in the case of wave filters [4], [16], and the lattice structures and the coupled-form biquad [9] to avoid zero-input limit cycles by adopting suitable arithmetic schemes, even under time-varying conditions. We now note that there exists an essentially similar controlled-rounding scheme which can completely suppress granular zero-input limit cycles and overflow oscillations for the model of Fig. 2 regardless of specific interconnections inside the $(N+1)$ pair.

The controlled rounding scheme to be adopted is based on the observation that the $(n+1)$-pair network $\mathscr{T}(n)$ is memoryless and does not, therefore, have loops (we would otherwise have delay-free loops). As a result, there is no unlimited bit-accumulation due to multiplications in $\mathscr{T}(n)$. We can therefore implement $\mathscr{T}(n)$ without any quantizer. This means firstly, that extra least significant bits generated by multiplications are not rounded off, and secondly, extra higher significant bits are retained instead of being allowed to overflow. Thus given $u(n)$ and $x(n)=$ $\left[x_{1}(n), \cdots, x_{N}(n)\right]^{t}$, the computation of $y(n)$ and $w(n)=$ $\left[w_{1}(n), \cdots, w_{N}(n)\right]^{t}$ is performed with full accuracy. Therefore, the following equation:

$$
\left[\begin{array}{l}
w(n) \\
y(n)
\end{array}\right]=\mathscr{T}(n) \cdot\left[\begin{array}{l}
\boldsymbol{x}(n) \\
u(n)
\end{array}\right]=\left[\begin{array}{ll}
\boldsymbol{A}(n) & \boldsymbol{b}(n) \\
\boldsymbol{c}^{t}(n) & d(n)
\end{array}\right]\left[\begin{array}{l}
\boldsymbol{x}(n) \\
u(n)
\end{array}\right]
$$

where the parameters $\boldsymbol{A}, \boldsymbol{b}, \boldsymbol{c}^{\boldsymbol{t}}, \boldsymbol{d}$ correspond to the usual state-space description, is implemented with full accuracy. Note that, in our developments the state-space parameters can, in general, be time varying.

The quantizers which are obviously needed, one per disjoint loop in order to avoid unlimited bit accumulation, are inserted before the delays. Thus

$$
x_{i}(n)=Q\left[w_{i}(n-1)\right]
$$

where $Q[\cdot]$ denotes the quantization operation. We assume fixed-point arithmetic. The quantization scheme to be adopted is "magnitude truncation" for rounding purposes. In addition, any overflow bit at the input of the quantizer is simply dropped and does not appear at the output of the quantizer. In this manner, we have

$$
\begin{aligned}
& x_{i}^{2}(n) \leqslant w_{i}^{2}(n-1) \\
& e_{i}^{2}(n) \triangleq w_{i}^{2}(n-1)-x_{i}^{2}(n) \geqslant 0
\end{aligned}
$$

and quantization always leads to loss of energy.

Now, under infinite precision arithmetic, in the absence of an input, (5) leads to

$$
w^{t}(n) w(n)-x^{t}(n) x(n) \leqslant-y^{2}(n)
$$

i.e.,

$$
\boldsymbol{x}^{t}(n+1) \boldsymbol{x}(n+1)-\boldsymbol{x}^{t}(n) \boldsymbol{x}(n) \leqslant-y^{2}(n) .
$$

Thus under infinite precision conditions, the internal energy always decreases, whenever $y(n)$ is nonzero, i.e., as long as a portion of the stored energy is "observable" at the output, it continues to decrease. But, in the limit as $n \rightarrow \infty$, it is not clear why $\boldsymbol{x}^{t}(n) \boldsymbol{x}(n)$ should not approach a nonzero lower bound. However, in practice, with finite wordlength arithmetic, if the quantization scheme described above is adopted, (13a) still holds, but instead of (13b) we get

$$
\begin{aligned}
\boldsymbol{x}^{t}(n+1) \boldsymbol{x}(n+1) & =Q\left[\boldsymbol{w}^{t}(n)\right] Q[\boldsymbol{w}(n)] \\
& \leqslant \boldsymbol{x}^{t}(n) \boldsymbol{x}(n)-y^{2}(n) .
\end{aligned}
$$

Thus as long as $y(n)$ does not become identically zero for all $n \geqslant$ some $N_{0}$, the result of the quantization (magnitude truncation) is to reduce the internal energy by a nonzero amount. Consequently, regardless of the initial energy, under zero-input conditions, the internal energy drops to zero after a finite number of iterations.

The two crucial factors involved in the suppression of limit cycles in the above manner are that (a) the output does not identically vanish for all $n \geqslant$ some $N_{0}$ when $\boldsymbol{x}^{t} \boldsymbol{x}$ is nonzero, and that, (b) the controlled rounding described above has been adopted. If these are satisfied, then the "instantaneous LBR or BR property" of $\mathscr{T}(n)$ ensures the rest. Condition (a), which is an observability requirement, corresponds to the "nonexistence" requirement of "degenerate sections" in the case of the Gray-Markel lattices [9]. Note that $\mathscr{T}(n)$ does not necessarily have to be lossy, for avoiding zero-input limit cycles.

In the case of the coupled-form biquad, the representation of Fig. 2 does not explicity show an output $y$ and an input $u$. Thus in our equations such as (13), the $y^{2}(n)$ term should be replaced with zero. However, if the coupled form poles are strictly inside the unit circle, the 2-pair structure is strictly lossy [9], and we have strict inequality in (13). Therefore, if the proposed arithmetic quantization scheme is adopted, the conclusions derived above remain valid.

Finally, it should be noted that, due to the quantization of the multiplier values in the matrix $\mathscr{T}(n)$, it may not be possible to ensure the orthogonality condition $\mathscr{T}^{t}(n) \mathscr{T}(n)=1$ in practice. However if the quantization of parameters is properly chosen, the quantity $\mathscr{T}(n)$ can be made lossy, i.e., $\mathscr{T}^{t}(n) \mathscr{T}(n) \leqslant 1$, and the conclusions of this section remain valid.

\section{Properties of Cascaded lBR Two-pair}

Consider now the cascade of LBR two-pairs as shown in Fig. 3. We call this the $\Pi$-cascade, to distinguish it from conventional multi-input multi-output cascade. It can be shown that a $\Pi$-cascade of LBR two-pairs is an LBR two-pair. Thus Fig. 3 is essentially an LBR two-pair constrained by the function $M_{0}(z)$. The normalized cascaded-lattice structures due to Gray-Markel are of this form where each two-pair has the transfer matrix

$$
\mathscr{T}_{m}(z)=\left[\begin{array}{cc}
k_{m} & \sqrt{1-k_{m}^{2}} \cdot z^{-1} \\
\sqrt{1-k_{m}^{2}} & -k_{m} z^{-1}
\end{array}\right]
$$

so that $\mathscr{T}_{m}^{t}\left(z^{-1}\right) \mathscr{T}_{m}(z)=\mathbf{1}$, and moreover, the constraining transfer function $M_{0}(z)=1$. In the case of cascaded 


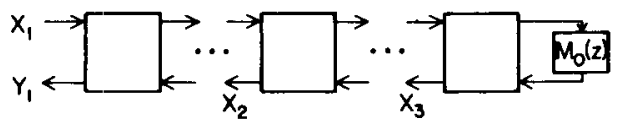

Fig. 3. The terminated LBR cascade.

wave digital filters, each two-pair can once again be considered LBR (if the external adders are scaled properly) but the constraining function $M_{0}$ is typically a constant with magnitude less than unity.

In this section, we derive certain properties of the structure of Fig. 3, without making specific assumptions about the forms of the two-pairs or $M_{0}(z)$, except that the two-pairs are LBR and that $M_{0}(z)$ is an LBR function. We do, however, assume that the structure is "minimal" or "canonic" in terms of the number of delays. Also, all transfer functions involved are assumed to be stable, under infinite-precision conditions. The derivations make extensive use of the paraunitary properties of digital LBR twopairs, listed in the Appendix.

Any LBR two-pair constrained by a scalar LBR also gives rise to a scalar LBR. So, if we are interested in $X_{2}$ in Fig. 3, we can redraw it as in Fig. 4, with $G_{0}(z)$ a scalar LBR function and $\mathscr{T}(z)$ an LBR two-pair with components $T_{i j}$. Define

$$
G(z) \triangleq \frac{Y_{1}(z)}{X_{1}(z)} \quad H_{3}(z) \triangleq \frac{X_{3}(z)}{X_{1}(z)}
$$

We now state and prove a number of properties of a constrained LBR two-pair:

Property 1: Orthogonality $-G(z)$ and $H_{3}(z)$ are orthonormal, ${ }^{3}$ i.e.,

$$
\left\langle H_{3}, G\right\rangle \triangleq \frac{1}{2 \pi j} \oint_{|z|=1} H_{3}\left(z^{-1}\right) G(z) z^{-1} d z=0
$$

provided, there is no direct (delay-free) path from $X_{3}$ to $Y_{1}$. Moreover, when $Y_{1}=X_{3}$, which happens if $T_{12}=T_{21}=$ $1, T_{11}=T_{22}=0$, the value of this integral is equal to unity.

Proof: Write $G(z)$ and $H_{3}(z)$ in terms of transfer matrix parameters

$$
\begin{aligned}
G(z) & =\frac{T_{11}(z)-\Delta(z) G_{0}(z)}{1-T_{22}(z) G_{0}(z)} \\
H_{3}(z) & =\frac{T_{21}(z) G_{0}(z)}{1-T_{22}(z) G_{0}(z)}
\end{aligned}
$$

where

$$
\Delta(z)=T_{11}(z) T_{22}(z)-T_{12}(z) T_{21}(z) .
$$

So $\left\langle H_{3}, G\right\rangle$ takes the form ${ }^{4}$

$$
\begin{aligned}
& \left\langle H_{3}, G\right\rangle \\
& \quad=\frac{1}{2 \pi j} \oint_{|z|=1} \frac{\tilde{T}_{21}(z) \tilde{G}_{0}(z)\left[T_{11}(z)-\Delta(z) G_{0}(z)\right] z^{-1}}{\left[1-\tilde{T}_{22}(z) \tilde{G}_{0}(z)\right]\left[1-T_{22}(z) G_{0}(z)\right]} d z .
\end{aligned}
$$

\footnotetext{
${ }^{3}$ Here $\langle\cdot, \cdot\rangle$ denotes an inner product.

${ }^{4}$ Superscript tilde stands for transposition followed by replacement of $z$ with $z^{-1}$
}

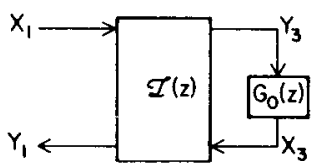

Fig. 4. Simplification of Fig. 3.

As such, it does not seem possible to proceed further because the exact forms of $T_{i j}$ and $G_{0}$ are not given. However, the constraints imposed by LBR properties lead to significant simplifications as shown next.

The numerator $N(z)$ of the integrand in (19) is

$$
N(z)=\tilde{G}_{0}\left[\tilde{T}_{21} T_{11}-\Delta \tilde{T}_{21} G_{0}\right] z^{-1} .
$$

Using the properties of LBR two-pairs given in Appendix A, we can show that

$$
-\Delta \tilde{T}_{21}=T_{12}
$$

and that

$$
\tilde{T}_{21} T_{11}=-T_{12} \tilde{T}_{22} .
$$

Thus (20) reduces to

$$
N(z)=\tilde{G}_{0} T_{12}\left[G_{0}-\tilde{T}_{22}\right] z^{-1}
$$

and since $G_{0}$ is LBR, we finally arrive at

$$
N(z)=T_{12}(z)\left[1-\tilde{T}_{22}(z) \tilde{G}_{0}(z)\right] z^{-1} .
$$

Consequently, (19) simplifies to

$$
\left\langle H_{3}, G\right\rangle=\frac{1}{2 \pi j} \oint_{|z|=1} \frac{T_{12}(z) z^{-1}}{1-T_{22}(z) G_{0}(z)} d z .
$$

With a change of variables, $z \rightarrow z^{-1}$ this becomes

$$
\left\langle H_{3}, G\right\rangle=\frac{1}{2 \pi j} \oint_{|z|=1} \frac{\tilde{T}_{12}(z) z^{-1}}{1-\tilde{G}_{0}(z) \tilde{T}_{22}(z)} d z
$$

Now, the poles of the integrand are at $z=0$, and at the points where

$$
1-\tilde{G}_{0}(z) \tilde{T}_{22}(z)=0
$$

is satisfied. But $T_{22}$ and $G_{0}$ are both BR functions and satisfy the inequality,

$$
\left|G_{0}\right| \leqslant 1 \quad\left|T_{22}\right| \leqslant 1
$$

outside the unit circle. So, the quantity on the left-hand side of (27) cannot be zero in $|z|<1$, unless both $G_{0}(z)$ the $T_{22}(z)$ are constants. But in view of realizability constraints, this is not possible.

In view of the minimality assumption, there is no pole-zero cancellation in (18). Moreover, by stability assumption, there cannot be an uncanceled zero of [1$G_{0}(z) T_{22}(z)$ ] on the unit circle. This means, essentially, that (27) does not hold at any point on the unit circle. Thus the integrand in (26) is analytic everywhere on the unit circle. Therefore, the only pole of the integrand inside the contour is at $z=0$. This gives

$$
\left\langle H_{3}, G\right\rangle=\frac{\tilde{T}_{12}(0)}{1-\tilde{G}_{0}(0) \tilde{T}_{22}(0)}=\frac{T_{12}(\infty)}{1-G_{0}(\infty) T_{22}(\infty)}
$$

and as there is no direct path from $X_{3}$ to $Y_{1}, T_{12}$ has a forward delay, so $T_{12}(\infty)=0$, proving (17). 


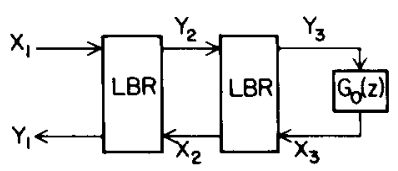

(a)

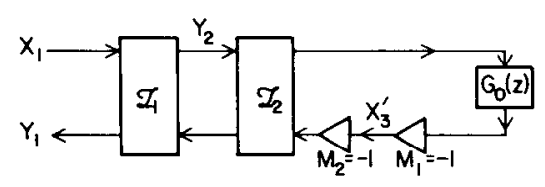

(b)

Fig. 5. (a) Generalization of orthonormality property. (b) Proving the generalization.

Next consider the case when $X_{3}$ and $Y_{1}$ are equal. Now, $T_{12}=T_{21}=1$ and by LBR property, $T_{11}=T_{22}=0$, hence from (29), $\left\langle H_{3}, G\right\rangle=1$. This completes the proof.

Note that the above sequence of arguments depends strongly upon the LBR nature of $\mathscr{T}(z)$ and $G_{0}(z)$. We now consider a generalized version of this property:

Consider now Fig. 5(a). Define

$$
H_{2}(z) \triangleq \frac{X_{2}(z)}{X_{1}(z)} \quad H_{3}(z) \triangleq \frac{X_{3}(z)}{X_{1}(z)} .
$$

Property 2: General Statement of Orthogonality

Unless there is a direct path from $X_{3}$ to $X_{2}, H_{3}(z)$ and $\mathrm{H}_{2}(z)$ are orthogonal:

$$
\left\langle H_{2}, H_{3}\right\rangle=0 .
$$

Proof: Define

$$
H_{2}^{\prime}(z) \triangleq \frac{X_{2}(z)}{Y_{2}(z)} \quad H_{3}^{\prime}(z)=\frac{X_{3}(z)}{Y_{2}(z)} \quad W(z)=\frac{Y_{2}(z)}{X_{1}(z)} .
$$

Hence,

$$
H_{2}(z)=H_{2}^{\prime}(z) W(z) \quad H_{3}(z)=H_{3}^{\prime}(z) W(z)
$$

and

$$
\begin{aligned}
\left\langle H_{2}, H_{3}\right\rangle & =\frac{1}{2 \pi j} \oint_{|z|=1} \tilde{H}_{2}(z) H_{3}(z) z^{-1} d z \\
& =\frac{1}{2 \pi j} \oint_{|z|=1} \tilde{H}_{2}^{\prime}(z) H_{3}^{\prime}(z) \tilde{W}(z) W(z) z^{-1} d z
\end{aligned}
$$

The quantity $\tilde{W}(z) W(z)$ is bounded on the unit circle (though not by unity) by, say, $K>0$. Then,

$$
\left\langle H_{2}, H_{3}\right\rangle \leqslant \frac{K}{2 \pi j} \oint_{|z|=1} \tilde{H}_{2}^{\prime}(z) H_{3}^{\prime}(z) z^{-1} d z=0
$$

by Property 1 . Now refer to Fig. 5(b). Here

$$
\frac{X_{3}^{\prime}}{X_{1}}=-H_{3}
$$

where $\mathrm{H}_{3}$ is the quantity appearing in the above equations. Clearly, the multiplier $M_{1}=-1$ can be incorporated into the constraining transfer function and the multiplier
$M_{2}=-1$ can be incorporated into the LBR two-pair $\mathscr{T}_{2}$. But $-G_{0}$ is still $\mathrm{LBR}$, and $\mathscr{T}_{2}$, thus modified, is still an LBR two-pair. Therefore,

$$
\left\langle H_{2}, H_{3}\right\rangle=-\left\langle H_{2}, H_{3}\right\rangle \leqslant 0 .
$$

Thus (35) and (36) imply (31) proving the property.

A third property we wish to consider is the following: Consider again Fig. 4, where an LBR two-pair $\mathscr{T}(z)$ is terminated by scalar LBR function $G_{0}(z)$. Define

$$
W(z)=\frac{Y_{3}(z)}{X_{1}(z)} .
$$

Property 3: Normalization- $W(z)$ is normalized in the $l_{2}$-sense, i.e.,

$$
\langle W, W\rangle \triangleq \frac{1}{2 \pi j} \oint_{|z|=1} \tilde{W}(z) W(z) z^{-1} d z=1
$$

even though the inequality, $|W(z)|<1$ may not hold for each $z$ on the unit circle.

Proof: We have

$$
W(z)=\frac{T_{21}(z)}{1-G_{0}(z) T_{22}(z)}
$$

hence,

$$
\begin{aligned}
\langle W, W\rangle & =\|W\|_{2}^{2} \\
& =\frac{1}{2 \pi j} \oint_{|z|=1} \frac{T_{21}(z) \tilde{T}_{21}(z) z^{-1}}{\left[1-G_{0} T_{22}(z)\right]\left[1-\tilde{G}_{0}(z) \tilde{T}_{22}(z)\right]} d z .
\end{aligned}
$$

Because of realizability constraints, we have a forward delay in $G_{0}(z) T_{22}(z)$, therefore,

$$
\left.\left[1-G_{0}(z) T_{22}(z)\right]\right|_{z=0}=\infty
$$

indicating that the pole at $z=0$ due to the $z^{-1}$ factor is cancelled. In view of the LBR properties of $\mathscr{T}(z)$ and $G_{0}(z)$, the only possible poles of the integrand in $|z|<1$ are solutions $z_{0}$ of

$$
1-G_{0}\left(z_{0}\right) T_{22}\left(z_{0}\right)=0
$$

i.e.,

$$
\tilde{G}_{0}\left(z_{0}\right)=\frac{1}{G_{0}\left(z_{0}\right)}=T_{22}\left(z_{0}\right) .
$$

and the quantity,

$$
\frac{T_{21}\left(z_{0}\right) \tilde{T}_{21}\left(z_{0}\right)}{1-\tilde{G}_{0}\left(z_{0}\right) \tilde{T}_{22}\left(z_{0}\right)}=\left.\frac{T_{21} \tilde{T}_{21}}{1-T_{22} \tilde{T}_{22}}\right|_{z=z_{0}}
$$

becomes, in view of LBR property,

$$
\left.\frac{1-T_{22} \tilde{T}_{22}}{1-T_{22} \tilde{T}_{22}}\right|_{z=z_{0}}=1
$$

So, the integral in (39) is the same as

$$
\frac{1}{2 \pi j} \oint_{|z|=1} \frac{z^{-1}}{1-G_{0} T_{22}(z)} d z
$$


A change of variables leads to

$$
\|W\|_{2}^{2}=\frac{1}{2 \pi j} \oint_{|z|=1} \frac{z^{-1}}{1-\tilde{G}_{0} \tilde{T}_{22}(z)} d z .
$$

So, the only pole to be considered is at $z=0$, and we have

$$
\|W\|_{2}^{2}=\frac{1}{1-G_{0}(\infty) T_{22}(\infty)}=1
$$

where, we have used the fact that $G_{0}(\infty) T_{22}(\infty)=0$ in view of realizability. A consequence of the above property is the following: Consider an all-pass filter with unity gain. If we extract a delay, the remaining two-pair can be assumed to be LBR without loss of any generality. We then have the situation shown in Fig. 4, with $z^{-1}$ in place of $G_{0}(z)$. Property 3 above shows that the "stored signal" 5 is scaled in $l_{2}$-sense. Note that Property 3 is generalization of the well-known scaling property of the normalized lattice [7].

None of the properties proved above require that the LBR two-pairs be reciprocal. The properties shown indicate that, in an LBR cascade as shown in Fig. 3, the external nodes are normalized in an $l_{2}$-sense. However, adders appearing inside each two-pair are not necessarily scaled.

\section{CONCLUding Remarks}

Some of the results derived in this paper have already been reported in the literature, by analyzing specific circuits in an independent manner. Thus in the case of wave filters [4], [16], the properties satisfied by various elements including the adaptors are individually analyzed, and then tied together in order to obtain many important conclusions. Similarly, the specific transfer matrices of the lattice two pairs are analyzed in [7], [9] in order to obtain properties satisfied by the cascaded lattice structures. In this paper, we obtain these and other conclusions, based only upon the paraunitary property, and do not assume anything further about the specific details of the circuit.

\section{APPENDIX A}

A digital two-pair (Fig.1) with inputs $X_{1}(z)$ and $X_{2}(z)$ and outputs $Y_{1}(z)$ and $Y_{2}(z)$ is described by its transfer matrix $\mathscr{T}(z)$ :

$$
\left[\begin{array}{l}
Y_{1} \\
Y_{2}
\end{array}\right]=\mathscr{T}(z)\left[\begin{array}{l}
X_{1} \\
X_{2}
\end{array}\right] \text { where } \mathscr{T}(z)=\left[\begin{array}{ll}
T_{11}(z) & T_{12}(z) \\
T_{21}(z) & T_{22}(z)
\end{array}\right]
$$

or equivalently by its chain matrix $\Pi(z)$ :

$$
\left[\begin{array}{c}
X_{1} \\
Y_{1}
\end{array}\right]=\Pi(z)\left[\begin{array}{l}
Y_{2} \\
X_{2}
\end{array}\right] \text { where } \Pi(z)=\left[\begin{array}{ll}
A(z) & B(z) \\
C(z) & D(z)
\end{array}\right]
$$

An LBR two-pair has a transfer matrix which satisfies the paraunitary property, i.e., $\mathscr{T}^{t}\left(z^{-1}\right) \mathscr{T}(z)=\mathbf{1}$ for all $z$.

\footnotetext{
${ }^{5}$ Signal stored in the memory element $z^{-1}$.
}

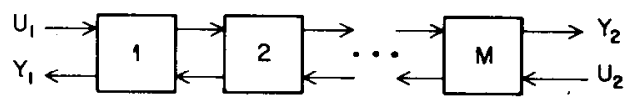

Fig. 6. An LBR cascade.

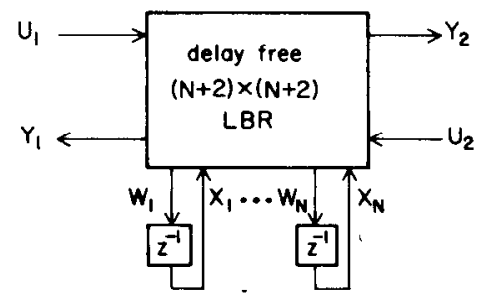

Fig. 7. The unified representation of the cascade of Fig. 6.

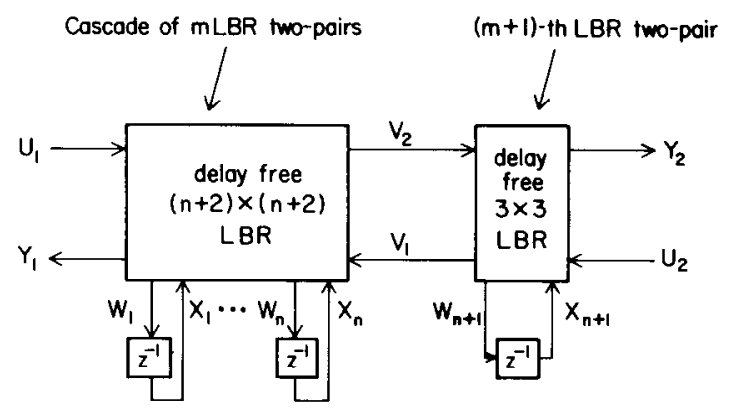

Fig. 8. Inductive proof of the representation.

In what follows, superscript " " stands for replacement of $z$ with $z^{-1}$, and corresponds to complex conjugation on the unit circle, $|z|=1$. The following properties hold for LBR two-pairs:

(i) $\quad T_{11} \tilde{T}_{11}=T_{22} \tilde{T}_{22}, \quad T_{12} \tilde{T}_{12}=T_{21} \tilde{T}_{21} \quad$ for all $z$.

In particular, on the unit circle, for an LBR two-pair, $\left|T_{11}\right|-\left|T_{22}\right|$ and $\left|T_{21}\right|=\left|T_{12}\right|$ for all steady-state frequencies $\omega$.

(ii)

$$
\begin{aligned}
\left|T_{i j}\right| \leqslant 1 & \text { if }|z| \geqslant 1 \\
\left|T_{i j}\right|<1 & \text { if }|z|>1 \text { unless } T_{i j} \text { is a constant } \\
1+B \tilde{B} & =A \tilde{A} \\
B \tilde{B} & =C \tilde{C} \\
\tilde{C}(A D-B C) & =B
\end{aligned}
$$

$$
\begin{aligned}
\tilde{C} D & =\tilde{A} B, \\
\tilde{C} A & =\tilde{D} B, \\
(A D-B C)(\tilde{A} \tilde{D}-\tilde{B} \tilde{C}) & =1, \\
A \tilde{A} & =D \tilde{D} .
\end{aligned}
$$

(Set (iii) is necessary and sufficient for paraunitariness.)

\section{APPENDIX B}

In this appendix, we justify the representation of Fig. 2. Consider the cascade of Fig. 6 where each two-pair is a "basic" LBR two-pair, i.e., LBR two-pair of order one or two. We first show that this cascade can be represented, as shown in Fig. 7, where all $N$ delays have been extracted, leaving behind an LBR $(N+2)$-port. (The number of delays $N$ in the cascade of Fig. 6 depends on the orders of the two-pairs, and is in the range $[M, 2 M]$.) The proof is 


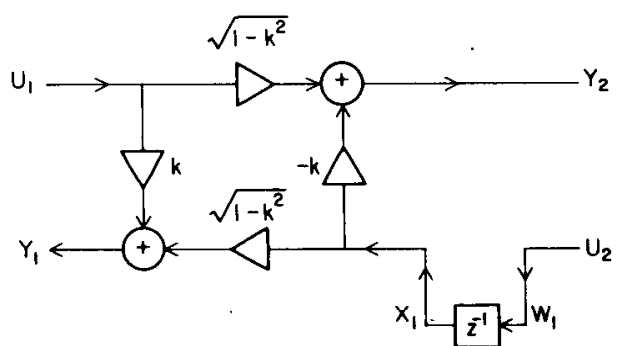

Fig. 9. The normalized lattice structure.

based on induction on the number of two-pairs in the cascade. Let us assume the representation of Fig. 7 to be valid for $M<m+1$. We then establish the validity for $M=m+1$. Fig. 8 depicts the situation, where the new $(m+1)$-th two pair is first-order LBR. By the induction hypothesis, for the cascade of $m$ LBR two-pairs, we have

$$
\begin{aligned}
& \left|U_{1}\left(e^{j \omega}\right)\right|^{2}+\left|V_{1}\left(e^{j \omega}\right)\right|^{2}+\left|X_{1}\left(e^{j \omega}\right)\right|^{2}+\cdots+\left|X_{n}\left(e^{j \omega}\right)\right|^{2} \\
& \quad=\left|Y_{1}\left(e^{j \omega}\right)\right|^{2}+\left|V_{2}\left(e^{j \omega}\right)\right|^{2}+\left|W_{1}\left(e^{j \omega}\right)\right|^{2}+\cdots\left|W_{n}\left(e^{j \omega}\right)\right|^{2}
\end{aligned}
$$

and for the $(m+1)$ th LBR two-pair, we get

$$
\begin{aligned}
\left|V_{2}\left(e^{j \omega}\right)\right|^{2} & +\left|U_{2}\left(e^{j \omega}\right)\right|^{2}+\left|X_{n+1}\left(e^{j \omega}\right)\right|^{2} \\
& =\left|V_{1}\left(e^{j \omega}\right)\right|^{2}+\left|Y_{2}\left(e^{j \omega}\right)\right|^{2}+\left|W_{n+1}\left(e^{j \omega}\right)\right|^{2} .
\end{aligned}
$$

From (A3) and (A4) wé get

$$
\begin{aligned}
\left|U_{1}\left(e^{j \omega}\right)\right|^{2}+\left|U_{2}\left(e^{j \omega}\right)\right|^{2} & \\
& +\left|X_{1}\left(e^{j \omega}\right)\right|^{2}+\cdots+\left|X_{n+1}\left(e^{j \omega}\right)\right|^{2} \\
= & \left|Y_{1}\left(e^{j \omega}\right)\right|^{2}+\left|Y_{2}\left(e^{j \omega}\right)\right|^{2} \\
& +\left|W_{1}\left(e^{j \omega}\right)\right|^{2}+\cdots+\left|W_{n+1}\left(e^{j \omega}\right)\right|^{2}
\end{aligned}
$$

which shows that the $(n+3) \times(n+3)$ delay-free circuit is indeed LBR. If the $(m+1)$ th two-pair is of second order, the proof is similar, except that resulting circuit is an $(n+4) \times(n+4)$ LBR.

It only remains to establish the basis for the induction. This is done by showing that all first- and second-order two-pairs under consideration have the representation of Fig. 7. For example, consider the first-order normalized Gray-Markel lattice structure (Fig. 9). The relevant $3 \times 3$ matrix is seen to be

$$
\left[\begin{array}{ccc}
k & 0 & \sqrt{1-k^{2}} \\
\sqrt{1-k^{2}} & 0 & -k \\
0 & 1 & 0
\end{array}\right]
$$

which is clearly LBR. The coupled form structure is known to be a lossy version of the normalized lattice [9], and hence leads to a BR rather than an LBR multiport. Next, consider a typical first order two-pair in a wave digital filter. It is shown in [10] that such two-pairs can be implemented in the form of Fig. 10, where $\mathscr{T}_{3}$ is LBR. Finally consider a typical second-order wave digital twopair. A minimal rcalization of such a two-pair can always be obtained with two-delays, and this realization can be

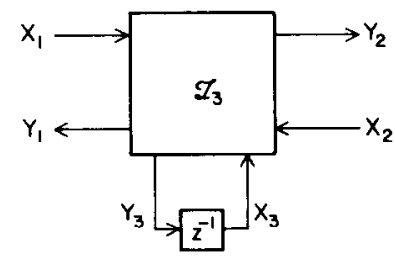

Fig. 10. The first-order wave two-pair.

redrawn as in Fig. 7 with $N=2$. One way to prove this is with the help of the discrete version of the "Lossless Bounded Real Lemma," discussed in [14]. The details are omitted here, in the interests of brevity.

The representation of Fig. 2 follows immediately from Fig. 7 because, in all the structures of interest, the input $U_{2}$ is constrained to be

$$
U_{2}=a \cdot Y_{2}
$$

where $|a| \leqslant 1$.

\section{REFERENCES}

[1] A. V. Oppenheim and R. W. Schafer, Digital Signal Processing. Englewood Cliffs, NJ: Prentice Hall, 1975.

[2] A. Fettweis, "Digital filter structures related to classical filter networks," Arch. Elek. Ubertrangung., vol. 25, pp. 79-81, Feb. 1971.

[3] M. N. S. Swamy and K. Thyagarajan, "A new type of wave digital filters," J. Franklin Inst., vol. 300, no. 1, pp. 41-58, July 1975.

[4] A. Fettweis, "Pseudopassivity, sensitivity and stability of wave digital filter," IEEE Trans. Circuit Theory, vol. CT-19, pp. 668-673, Nov. 1972.

[5] P. DeWilde and E. Deprettere, "Orthogonal cascade realization of real multiport digital filters," Int. J. Circuit Theory Appl., vol. 8, pp. $245-277,1980$

16] A. H. Gray, Jr. and J. D. Markel, "Digital lattice and ladder filter synthesis," IEEE Trans. Audio Electroacoust., vol. AU-21, pp. 491-500, Dec. 1973

[7] "A normalized digital filter structure," IEEE Trans. Acoustics, Speech, Signal Processing, vol. ASSP-23, pp. 268-277, June 1975

[8] D. A. Vaughn-Pope and L. T. Bruton, "Transfer function synthesis using generalized doubly terminated two-pair networks." IEEE Trans. Circuits Syst., vol. CAS-24, pp. 79-88, Feb. 1977.

[9] A. H. Gray, Jr., "Passive cascaded lattice digital filters," IEEE Trans. Circuits Syst., vol. CAS-27, pp. 337-344, May 1980.

[10] P. P. Vaidyanathan and S. K. Mitra, "Low passband sensitivity digital filters: A generalized viewpoint and synthesis procedures," Proc. IEEE, vol. 72, pp. 404-423, Apr. 1984

11] P. P. Vaidyanathan, "A general theory and synthesis procedure for low sensitivity digital filter structures," Ph.D. dissertation, Dep. of Electrical and Computer Engineering, Univ. of California, Santa Barbara, June 1982.

[12] R. W. Newcomb, Linear Multiport Synthesis. New York: McGraw-Hill, 1966.

[13] V. Belevitch, Classical Network Synthesis. San Francisco, CA: Holden-Day, 1968.

[14] B. D. O. Anderson and S. Vongpanitlerd, Network Analysis and Synthesis, Englewood Cliffs, NJ: Prentice Hall, 1973.

[15] C. W. Barnes and A. T. Fam, "Minimum norm recursive digital filters that are free of overflow limit cycles," IEEE Trans. Circuits Syst., vol. CAS-24, pp. 569-574, Oct. 1977.

[16] A. Fettweis and K. Meerkotter, "Suppression of parasitic oscillations in wave filters," IEEE Trans. Circuits Syst., vol. CAS-22, pp. 239-246, Mar. 1975.

[17] B. Friedlander, "Lattice filters for adaptive processing," Proc. IEEE, vol. 70, pp. 829-867, Aug. 1982.

[18] S. K. Mitra, P. S. Kamat, and D. C. Huey, "Cascaded lattice realization of digital filters," Int. J. Circuit Theory Appl., vol. 5, pp. 3-11, 1977.

[19] H. W. Schuessler, "A stahility theorem for discrete systems," IEEE Trans. Acoust., Speech, Signal Processing, vol. ASSP-24, pp. 87-89, Feb. 1976.

[20] A. Fettweis. "Some general properties of signal flow networks." in Network and Signal Theory, (Eds. J. K. Skwirzynski and J. O. Scanlan), London, England: Peter Peregrinus, 1973. 


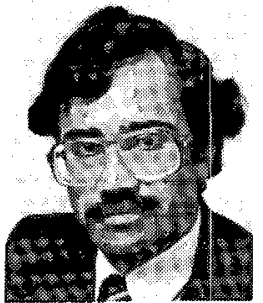

P. P. Vaidyanathan (S'80-M'83) was born in Calcutta, India, on October 16, 1954. He received the B.Sc (hons.) degree in physics, and B. Tech. and $M$. Tech degrees in radiophysics and electronics from the University of Calcutta, India, in 1974, 1977, and 1979 respectively, and the Ph.D. degree in electrical and computer engineering from the University of California, Santa Barbara, in 1982.

He was a Post-Doctoral fellow at the University of California, Santa Barbara, from September 1982 to February 1983. Since March 1983, he has been with the California Institute of Technology, Pasadena, as an Assistant Professor of Electrical Engineering. His main research interests are in digital signal processing, linear systems and filter design. He served as the Vice-Chairman of the Technical Program Committee of the 1983 IEEE International Symposium on Circuits and Systems.

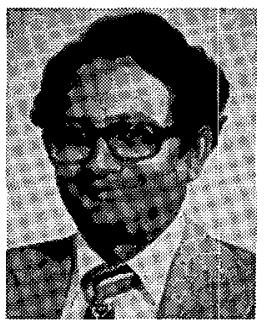

Sanjit K. Mitra (S'59-M'63-SM'69-F'74) received the B.S. (hons.) degree in physics from the Utkal University, India, in 1953, the M.Sc. (Tech.) degree in radio physics and electronics from the University of Calcutta, India, in 1956, the M.S. and $\mathrm{Ph} . \mathrm{D}$. degrees in electrical engineering from the University of California, Berkeley, in 1960 and 1962, respectively.

He was a member of the faculty of the Cornell University, Ithaca, NY, from 1962 to 1965 , and a member of Technical Staff of the Bell Laborato- ries from 1965 to 1967 . He joined the faculty of the University of California, Davis, in 1967 and transferred to the Santa Barbara (UCSB) campus in 1977 as a Professor of Electrical and Computer Engineering. From July 1979 to June 1982 he served as the Chairman of the Department of Electrical and Computer Engineering at UCSB. He is a consultant to the Lawrence Livermore National Laboratory, Livermore, CA, and the Los Alamos National Laboratory, Los Alamos, NM, and is the Consulting Editor for the Electrical/Computer Science and Engineering Series of the Van Nostrand-Reinhold Company, NY. He has also served as a consultant to Ampex Corporation, Fairchild Semiconductor, Siliconix, HDR Sciences, the U.S. Army, General Motors Delco Division and the World Bank. He has held visiting appointments at the Indian Institute of Technology, New Delhi; Kobe University, Japan; University of Erlangen-Nuernberg, West Germany, the Australian National University, Canberra; Tampere University of Technology, Tampere, Finland, and the Federal University of Rio de Janeiro, Brazil. He has published a number of papers in active and passive networks, and digital filters, and is the author of Analysis and Synthesis of Linear Active Networks and An Introduction to Digital and Analog Integrated Circuits and Applications, and Editor of Active Inductorless Filters, Co-Editor of Modern Filter Theory and Design (with G. C. Temes), Co-Editor of Two-Dimensional Digital Signal Processing (with M. P. Ekstrom). He holds two patents in active filters. He was an Associate Editor of the IEEE Transactions on Circuits and Systems, and served on the editorial boards of the ProCEEDINGS OF THE IEEE and IEEE Press, and presently is on the editorial board of Circuits, Systems and Signal Processing. He has been a member of the Administrative Committee of the IEEE Circuits and Systems Society, Local Arrangements Chairman (1969), General Chairman (1974), and Program Chairman (1983) of the annual IEEE International Symposium on Circuits and Systems. He is the recipient of the 1973 F.E. Terman Award of the American Society of Engineering Education, a Visiting Professorship from the Japan Society for Promotion of Science in 1972, and Senior Fulbright Award for Brazil in 1984

Dr. Mitra is a Fellow of the American Association for the Advancement of Science and a member of the American Society for Engineering Education, Sigma Xi, and Eta Kappa Nu. 